\title{
INTERACTIVE SPATIAL WEB-APPLICATIONS AS NEW MEANS OF SUPPORT FOR URBAN DECISION-MAKING PROCESSES
}

\author{
E. Gebetsroither-Geringer ${ }^{1}$, R. Stollnberger ${ }^{1}$, J. Peters-Anders ${ }^{1}$ \\ ${ }^{1}$ AIT, Austrian Institute of Technology, Giefinggasse 4, 1210 Vienna, Austria - (ernst.gebetsroither, romana.stollnberger, jan.peters- \\ anders)@ait.ac.at
}

KEY WORDS: Geo-visualization, Interactive Web-Applications, Spatial Decision Support Systems, R/Shiny Framework

\begin{abstract}
:
Citizen participation, co-creation -a joint development of professionals and citizens- initiatives for urban planning processes have increased significantly during the last few years. This development has been strongly supported by the evolution of Information and Communication Technologies (ICT). E.g., it has never been easier to get information through your mobile devices wherever and whenever you want it. Public open spatial data is available in many cities around the world and web-based applications use this data to provide tools and services for many different topics such as traffic information, or the communication of health-related information (e.g. ozone, particulate matter or pollen loads). This paper presents typical problems of such web-applications in terms of application design and implementation and usability evaluation via describing three case study applications which have been developed recently. It tries to answer the question: How can this kind of geo-services be developed and used by scientists to enable public participation within data gathering and urban planning processes? All three applications have the common goal to provide interactive geovisualization and analysis features which are tailored to support users in their urban planning processes. The innovation of those applications lies in their flexibility regarding the topics they can tackle and their capability to perform interactive analyses triggered by the user. The applications have been built with a strong focus on exploring the available data (e.g. Open Government Data - OGD). Two of the applications have been implemented using the R-Shiny framework, the third application, the smarticipate platform, has been developed using ReactJS for the front-end, running a MongoDB in the background which is fed via a micro-service framework. In the latter application, the users can configure topics, i.e. the platform enables the user to create new services for different planning issues.
\end{abstract}

\section{INTRODUCTION}

\subsection{Background}

During the last few years the active involvement of citizens in urban planning processes increased significantly. The idea behind urban co-creation is to create a connection between professionals (urban planners, decision-makers) and citizens and to intervene, participate and engage with each other to transform the urban environment, regardless of the social or professional background of the participants (Dörk and Monteyne, 2011). Common participation was strongly facilitated through the evolution of ICT. (Mueller et al., 2018) present Citizen Design Science as a new strategy for cities to integrate the citizens' ideas and wishes into the urban planning process. The idea is to combine crowdsourcing methods through modern ICT with active design tools. The development of web-based IT-tools enables citizens to easily take part in participatory city planning processes (Khan et al., 2014). Most participatory planning processes happen at a very local level, e.g. on a neighbourhood or district level thus information on this level is necessary to engage with the people. Because of this, new participatory tools often need to incorporate spatial data in high resolution and decision-making features tailored to the users. The combination of geographic information systems (GIS) functionalities such as spatial data management and cartographic display with webbased Spatial Decision Support Systems (SDSS) providing flexible user interfaces and analytical modelling capabilities enable the participation of users in planning processes (Sugumaran and Sugumaran, 2007). GIS alone cannot solve the problems of planning processes but integrating additional ICT tools seems to offer the digital infrastructure for developing decision making tools (Voss et al., 2004).
Additionally, the availability of spatial Open Government Data (OGD) increased significantly during the last years. However most often these data are used by experts, only, e.g. within research projects because other stakeholders do most often not know how to use those datasets.

\subsection{Problems}

In the past, Spatial OGD has often been used to create services to support individual spatial planning decisions. Most often these services (tools) have been developed as a static web service using HTML5/Javascript programming and Web Map Servers to visualize different pre-processed results. Nonetheless, the development of more appropriate services, which enable the user to do their own analyses, are not very common. One problem to engage with local stakeholders are often static visualizations of pre-processed results as the stakeholders want to have more specific - i.e., tailored to their needs - results. Thus, there is a clear need to create this kind of services and ideally the created services should be self-explanatory and "all-rounder" programs, i.e. programs which are easy to use and understand. An additional problem is that these services often lack a good business model and thus the development is often done in funded research projects with time and budget constraints which allows neither for intensive development, nor for improvement cycles throughout the development process.

Another crucial topic all applications, tools or services must deal with, is user engagement. Here it is important to provide the content in a way the users can understand and relate it to their environment, meaning that engagement is much easier if it really tackles the stakeholders' interests. Design guidelines for webapplications supporting user engagement e.g. exist since almost 20 years. Wroblewsky and Rantanen mentioned already in 2001 
twelve specific design guidelines (Wroblewski and Rantanen, 2001), Fowler and Stanwick a few years later (Fowler and Stanwick, 2004). Web technology innovations in the last few years allow software designers with the help of feature rich frameworks such as e.g. jQuery, AngularJS, Bootstrap, React and Node.js to quickly develop responsive mobile-friendly applications (Shahzad, 2017). Several challenges in developing web-applications must be tackled, e.g., to deliver simplicity and intuitivity as the internet users are not very patient and might move on otherwise. Another challenge is the look and feel of an app to ensure a flawless user experience which goes hand in hand with another criteria for success, the performance of the application and its scalability (Prat, 2018).

The following paper presents an introduction to web-based applications including use cases, target groups and software design issues as well as usability evaluation. Therefore, we will depict three case studies dealing with the above described problems and approaches for solutions with a special focus of spatial applications.

\section{WEB-BASED APPLICATIONS TO SUPPORT URBAN DECISION MAKING}

\subsection{Use cases}

There are various types of goals web-based applications are addressing. Some aim to inform citizens about a particular problem, raise awareness or visualize different topics. Other applications aim at identifying current problems in local communities, providing citizens an opportunity to discuss daily issues as well as providing a platform to voice their opinion and directly get in touch with responsible city stakeholders. (Desouza C., Kevin and Bhagwatwar, Akshay, 2012). In summary, the following main types can be classified.

Applications that:

- inform, create awareness, increase transparency.

This can be e.g. displaying information about the traffic situation, on health issues (ozone, fine dust or pollen loads) via e.g. maps showing pre-calculated results. Usually, these applications contain top-down created information, aiming at providing content related to the personal environment of the users, but often they lack specific (spatial) data the users would need to take informed decisions.

- identify, report current problems.

Here, problems like e.g. damaged infrastructure (e.g. pot-holes), or crammed waste bins are reported, most often to the municipal administration. Sometimes these applications are more elaborated, enabling to start a discussion about the problem and its possible solutions within a forum. This represents a bottom-up process and user engagement is rather easy as the problems are linked to the personal environment of the users.

- $\quad$ support mid to long term planning processes.

In order to enable interactive analyses supporting mid to long term decision making (planning) processes, technologies which facilitate participation through interactive analysis and mapping tools are useful in two ways: On the one hand, urban planners and city authorities better understand challenges citizens are facing in their neighbourhoods. On the other hand, users/affected population within the investigated urban area can - via the use of interactive elements - try out the effects of various scenarios for themselves and thus are motivated to change inappropriate circumstances as they wish.

The diversity of topics that can be addressed through web-based applications is quite high. Possible topics are:

- public transportation (real-time information on public transport)

- $\quad$ public services (report problems/infrastructure issues, dangerous road situations, pollution in the streets, etc., (example applications: "Sag's Wien" in Vienna (Stadt Wien, 2018) or Improve My City (URENIO Research, 2018)),

- public safety (e.g. create awareness about crime incidents with map visualization of any crime incidents),

- $\quad$ spatial planning/citizen participation or

- urban, energy planning (e.g. the digital thematic city map "Wiener Umweltgut" (Vienna Environmental Goods (Wiener Umweltschutzabteilung, 2018)) provides access to environmentally relevant information like e.g. the solar potential of roof surfaces, the waste heat potential of production sites or the position of protected landscape areas.

Designing and planning urban areas is a complex task especially addressing the variety of challenges such as energy and climate topics, transport, public safety and high crime rates, social inequality, population growth in cities and health issues. Only recently, more specific tools and services for designing and planning urban areas emerge ("DeCodingSpaces Toolbox," 2018, "UrbanFootprint," 2018, "UrbanSim Inc," 2018; Gebetsroither-Geringer and Loibl, 2014)

\subsection{Target users and how to mobilize them}

One of the greatest challenges concerning user engagement is encouraging citizens to get involved. The complexity of spatial planning problems requires the competency of experts in different fields like urban planners, mobility/climate experts as well as local stakeholders (city authorities, citizens) for defining appropriate development scenarios.

Bringing relevant sources of information together must involve supportive policies and governance platforms who promote the development of applications that use open data to trigger change at the local level. Governments can promote citizen empowerment and allow citizens to frame solutions for local problems by making the right set of tools available to them. By providing an assortment of incentives, e.g. prizes and competitions, the share of participants can even be increased (Desouza C., Kevin and Bhagwatwar, Akshay, 2012).

Another possible way to mobilize (motivate) users is to use the gamification approach. The idea is to use game design elements in non-game contexts (Deterding et al., 2011). Gamification is a multi-validated approach to increase motivation and willingness to participate in participative processes. E.g. citizens can collect engagement points whenever their ideas for a new urban planning area get upvoted by fellow citizens (Van Ransbeeck, Wietse, 2016).

Gamification has already been successfully used in various application areas to promote participation, for example in the context of civil courage (Coronado Escobar and Vasquez Urriago, 2014) civic participation (Thiel, Sarah-Kristin and Lehner, Ulrich, 2015) e-learning (Barata et al., 2013) and e- 
government (Al-Yafi and El-Masri, 2016). Also, in the field of mobility the application of gamification has produced positive results, e.g. in relation to the promotion of sustainable forms of mobility (Kazhamiakin et al., 2015).

\subsection{Usability evaluation}

Usability is considered to be one of the most important quality factors for either success or failure of web-applications ((Fernandez et al., 2011), (Mvungi and Tossy, 2015), (Cayola and Macías, 2018)). (Nielsen, Jakob, 2012) clearly state if a website is difficult to use or the information is hard to read, people leave, because "There's no such thing as a user reading a website manual". The term usability was derived from the term "user friendly' and is defined as "the extent to which a product can be used by specified users to achieve specified goals with effectiveness, efficiency and satisfaction in a specified context of use" (international standard ISO 9241, covering ergonomics of human-computer interaction (Matera et al., 2006).

Due to the complexity and variety of web based applications that evolved during the last years, many methods, techniques, and tools with which to address and evaluate web usability issues have been developed. In order to ensure user satisfaction, an evaluation to assess the application functionality, to verify the effect of its interface on the user, and to identify any specific problem with the application should be conducted. Depending on the phase, in which evaluation is performed, there are two possibilities: either formative evaluation (takes place during design), or summative evaluation (to be done for final evaluation after the product has been developed). The most common methods are user testing, where the real users are studied, usability inspection, where specialists evaluate and web usage analysis, where the users' behaviour is studied via access statistics (e.g. Google Analytics). The method of user testing is quite extensive due to a very time-consuming process and required personnel. Therefore, usability inspection methods emerged where developers and usability experts predict usability problems that could be detected through users testing. E.g. in a heuristic evaluation, experts analyse the application against a list of recognized usability principles - the heuristics. Cognitive walkthroughs, on the other hand, simulate the steps the users will take in specific situations of use and why. An expert evaluator or a group of evaluators walk through a series of tasks to be performed on the interface and discuss usability issues in order to understand if users would know what to do (Matera et al., 2006).

\section{INTERACTIVE GEO-VISUALIZATION AND ANALYSES}

\subsection{Application design}

One of the key questions regarding the design of interactive web services is: For whom are they developed, with what objective and which purpose? For services (tools) aiming to be used by different stakeholders (urban planners, GIS-experts, citizens etc.) with different knowledge, this would imply a huge amount of effort and a direct interaction with the users during the development phase. Professional software development companies like Google, Microsoft or Apple e.g., devote a huge amount of their effort in usability testing and beta-testing applications. Recently, a trend has emerged that more and more web services are being developed by data scientists, instead of software development experts. Development frameworks like RStatistic and Shiny (see chapter 3.2) enable to build web applications for interactive geo-visualization and analyses with low effort. The innovative approach is that these applications enable interactive, user driven analyses including data collection.

In the following, two different implementation approaches are discussed which have been used in the case studies in this paper. Both tackle the problems discussed above in a slightly different manner. The first approach tries to create small, tailor made applications integrating the users' needs and requirements whereas the second tries to develop a platform (a middleware) enabling to configure tailored services for manifold topics in urban planning.

\subsection{Implementation approach using the R/Shiny Framework}

Shiny (R-Studio, 2018) is an R Package (a so-called library) to build interactive web-applications with a rapidly increasing user community, providing code examples via a gallery of showcases. This has the advantage that applications can be built with relatively low effort. The applications can be embedded into RMarkdown documents. Shiny applications can use CSS themes, HTML widgets and pure JavaScript. What Shiny makes so powerful is that it is part of $R$ (R Foundation, 2018) and within the last decade $\mathrm{R}$ became more recognized to be used for geocomputational issues (Lovelace et al., 2018). Another important aspect is, that the R/Shiny combination can be used to build webapplications, which $\mathrm{R}$ as well as Shiny are able to run, either on developer owned servers or on a dedicated (cloud) server owned by R-Studio, the developer of Shiny. A clear focus of this approach is to use pre-developed software parts, either of Shiny or R, as much as possible, thus the developers can concentrate on their data analyses, i.e. the tailored information for the user, which can be interactively calculated for the special needs of the users.

\subsection{Implementation using a Configuration Approach via a Middleware}

In comparison, the smarticipate platform $\left({ }^{1}\right)$ described in chapter 4.3, has been developed using ReactJS for the frontend, running a MongoDB database in the background which is fed via microservice framework which is handling the user management, data storage and communication channels of the platform. Deviating to the above described implementation approach, the smarticipate platform is an already developed generic platform which enables the users to create topics and new services for participatory processes and different planning questions.

\subsection{Usability improvement and evaluation}

As already mentioned above, an application (i.e. a software) developed by data scientists cannot be developed with the same economical effort as e.g. business software or computer games, since the budget for the programming is usually much lower than in commercial developments. Nevertheless, the usability should follow standard development rules mentioned in section 1.2 and e.g. use tooltips to explain the use of certain features. Online manuals, including annotated screenshots and video tutorials, can be easily created e.g. by using tools like HelpNDoc (IBE Software, 2018).

For the smarticipate case, important usability improvements have been introduced by optimizing the platform for desktop and

https://www.smarticipate.eu/platform/, last accessed 14.05 .2018 
mobile use, as well as programming the frontend in HTML5 which makes it platform independent, since it runs in any browser in any operating system. Using R/Shiny does not support mobile use out of the box, meaning the development responsive UI's regarding the used device.

\subsection{Data Quality}

Regarding the used datasets, the availability of OGD increased significantly in the last few years. This has also led to qualitative differences, like incompleteness of data-sets or data-sets being not up-to-date or incorrect. To work with those data, it is usually necessary to check and validate them or let them be checked and validated by experts in advance before using them in webapplications. Data validation is also a crucial point when users can enter their own data e.g. (see also 4.2.3).

\subsection{Performance}

According to (Sugumaran and Sugumaran, 2007) performance is one of the biggest limiting factors in the development of web based SDSS. Those limitations arise mostly from the use of large raster and vector data-sets and the need to also move large spatial objects between the server and the client via network connections.

Regarding performance, the programming language $\mathrm{R}$ also provides room for improvement (Martin, 2016). This is especially the case if no effort is spent on overcoming the performance bottleneck of $\mathrm{R}$ in the future, but already several techniques exist regarding those issues (Lim and Tjhi, 2015). One of these is to use external tools such as gdal (GDAL/ORG contributors, 2018) or QGIS connected to R, to increase the performance within R-scripts and Shiny web-applications. Nevertheless, it is still an important issue of many if not all webapplications that users will not spend a long time waiting for results especially if they do not get any feedback (performance bars, explanation dialogs etc.) during the processing on the server.

\section{Case Study Applications}

The following case study applications have been developed within different projects and use the above described implementation approaches. Their main purpose is to show - as a proof of concept - how this kind of geo-services can be developed and used by data scientist to enable public participation within data gathering and urban planning processes.

\subsection{PV-Potential Calculator}

The PV-Potential Calculator is an interactive web-based service enabling geo-visualization and interactive analyses.

\subsubsection{Background and Motivation}

The PV-Calculator has been developed within the Syn[En]ergy $\left(^{2}\right)$ project. Syn[En]ergy investigated, via an inter- and transdisciplinary approach, synergy and conflict potentials of photovoltaics in open urban areas, with other use demands for the open areas. The project developed a typology and practical solutions for selected areas with regards to requirements covering economical, urban planning and design, legal as well social aspects. Those requirements were then evaluated by stakeholders from enterprises, the city administration and citizens.

\footnotetext{
${ }^{2}$ http://synenergy.boku.ac.at/ , last accessed 14.05.2018
}

Regarding the history of the tool it is important to note that the tool had never been planned to be developed within the project in the first place, but the aim was to develop an application to demonstrate the photovoltaic potential of different urban open spaces like parking lots, playgrounds or parks etc. around Vienna, based on the available open spatial data from different sources. Problems arose during the detailed data analyses where the project was facing poor data quality regarding the exact georeference of the required data of urban open spaces. Compared to standard solar cadastre maps, this application is thus more innovative as it enables the users to calculate potentials for their own areas of interest.

\subsubsection{Application design and Implementation}

The UI - a standard dashboard layout - was created using the Shiny package of straight from R. With this package, a simple but quite intuitive interface - following standard web design rules - can be created without huge development effort and therefore more effort can put into the functionality of the application mainly using $\mathrm{R}$ as programming framework. Figure 1 shows a screenshot of the PV-Calculator. Four simple steps have been designed to enable the user to select an area, for which the PV potential should be calculated. Figure 2 shows the result after the calculation of the area. Besides the calculation of specific values for a selected area, the economic potential is roughly evaluated.

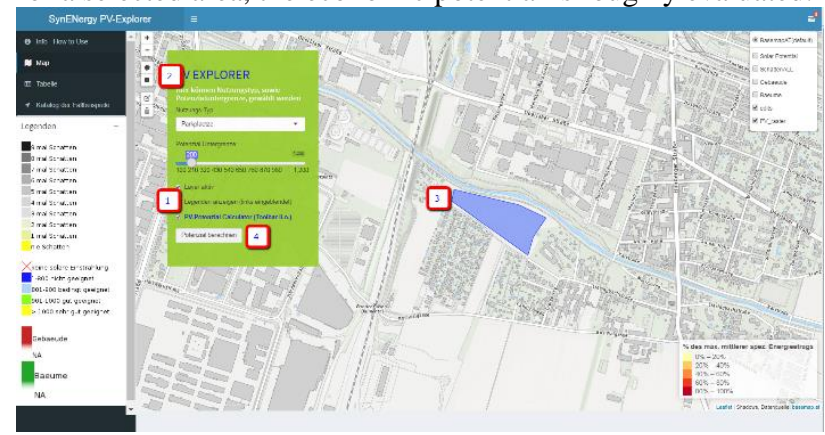

Figure 1. PV-Potential Calculator-Edit @ AIT 2018

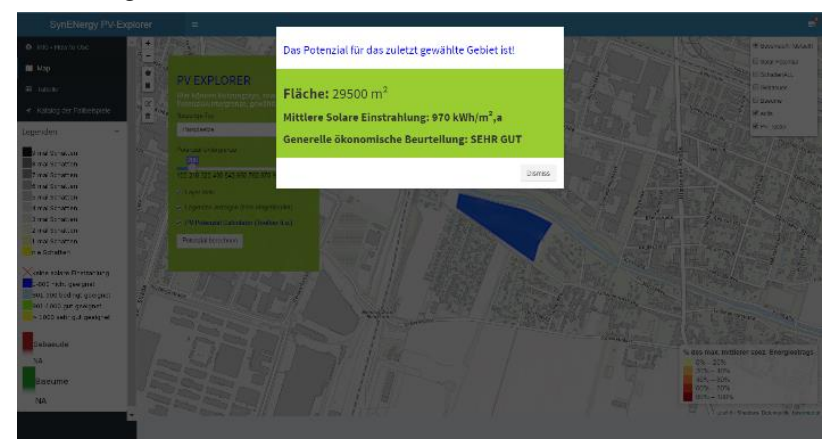

Figure 2. PV-Potential Calculator-Results @ A ATT 2018

\subsubsection{Difficulties and possible solutions}

The following Figure 3 shows a message which is triggered by an incorrect usage meaning that the selected area contains buildings, which would lead to a wrong analysis result. Nevertheless, that this main incorrect usage (selectin areas with buildings) has been inhibited, it is clear that not all similar problems can be solved in such kind of service. Thus, an introduction how to use this service correctly is important. Annotated screenshots and short tutorial videos have been developed as an extensive documentation or long user manuals would not work in this context. Regarding the performance of the application, the most demanding step - the calculation of the PV- 
potential in a resolution of $1 \mathrm{~m}^{*} 1 \mathrm{~m}$ for the entire area of Vienna - was already done within the Syn[En]ergy project. For a small selected area calculation times of a few seconds occur and a progress bar provides feedback to the user during this process. If a huge area would be selected it would take very long to get the results, but in the context of this application it is very unlikely that this is a serious PV-installation scenario.

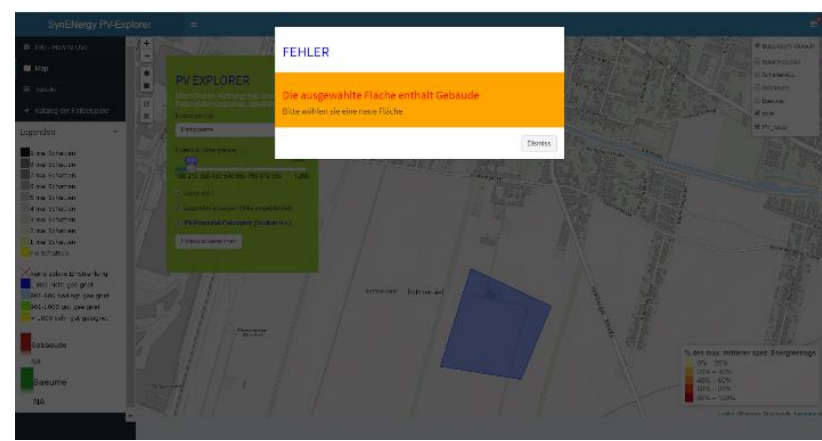

Figure 3. PV-Potential Calculator-User Guide (incorrect usage) (C) AIT 2018

Usability tests have not been conducted extensively, but within the project Syn[En]ergy team tests have been made by using a mix of methods (user testing and heuristic evaluation). The application can be used on devices up to the size of 8 " displays quite well but is built for larger ones.

\subsection{Waste Heat Explorer}

This web-based service is an example for using interactive geovisualization for data gathering and analyses.

\subsubsection{Background and Motivation}

The "Waste Heat Explorer" is part of the "Memphis" project which aims at creating a methodology to evaluate and map the potential of waste heat from the industry, the service sector or from sewage water by using internationally available open data. The project addresses this issue through the analysis of low-grade and spatially distributed heat potentials. The project Memphis is still ongoing, but a prototype of the web application "Waste Heat Explorer" is already available. The motivation for its development was (i) to provide interested stakeholders a possibility to analyse calculated waste heat potentials from industry on a map, (ii) to provide a web-based opportunity to add new possible waste heat sources by the users (data gathering) and (iii) to evaluate the already calculated potentials with the help of local experts or members from the industry or service sector.

\subsubsection{Application design and Implementation}

The following Figure 4 shows a screenshot of the designed web application. Figure 4 depicts in comparison to Figure 1 the similarity of the UI which is based on the development with the same framework R and Shiny. Nevertheless, this web application provides a very different feature to gather data regarding possible sources for waste heat. The user can, with five very simple steps, add a new location with company name, industrial sector affiliation, number of employees and address, together with privacy status assignment. The application uses the developed algorithm in the background to calculate the potential out of the input information and shows the results afterwards (Figure 5). The users have then the opportunity to overwrite the precalculated results. This application has beyond the input of new sources further features. The application records when a user overrules the pre-calculated results and this information can be used to evaluate the current calculation method developed during several projects.

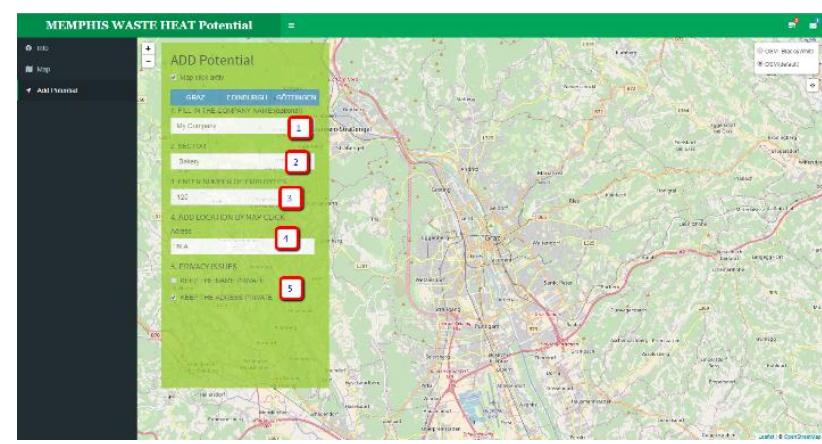

Figure 4. Waste Heat Explorer - Add Potential @ AIT 2018

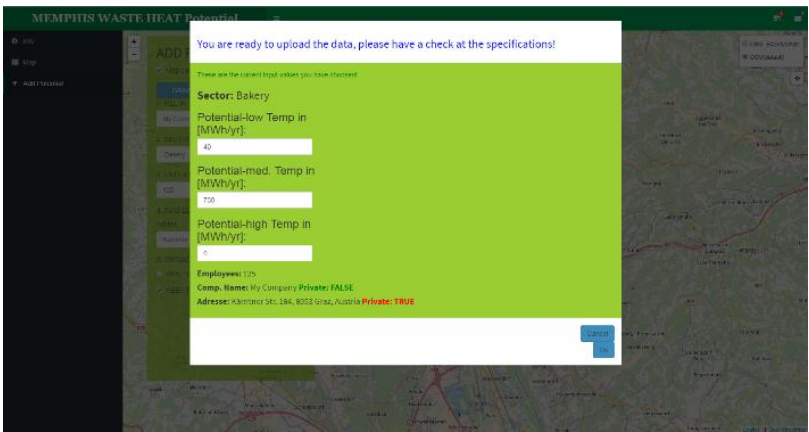

Figure 5. Waste Heat Explorer - User Interactions (C) AIT 2018

\subsubsection{Difficulties and possible solutions}

Smaller difficulties as verifying the address or to make sure that all necessary content is filled in before the calculation starts, thus the calculation does not throw an error, exist. These difficulties can be solved with rather small effort. A main problem all these user driven data gathering application have in common is how to evaluate the data entered by the user. One could just for fun indicate a source with wrong information leading e.g. to a significant waste heat potential, at a location where it does not exist. Solutions for this are not that easy. One could think of authorisation (e.g. with a login and personal identification) is a possible mean to solve this, but this most probably will decrease the willingness of people to participate in the data gathering procedure. The "Waste Heat Explorer" can find out outliers which afterwards can be further verified. The correction by the general public could be used as another possible way to find this kind of "fraud", as in Wikipedia. Serious games used for data collection can implement such kind of control mechanism rather easily as points for players verifying the data input from other players. However, the Waste Heat Explore does not include these methods, but it is assumed that no high incentives exist to enter wrong information. Regarding the performance, this application is on the save side, but the UI also lacks usability testing and should not be used on small devices.

\section{3 smarticipate}

\subsubsection{Background and Motivation}

Project smarticipate's aim is to develop a web-platform which can be used as a participatory citizen dialogue system, meaning it should provide a means of e.g. transforming OGD into new forms of transparency during planning processes within a city. The platform should be flexible and generic to provide the basis for all kinds of different topics to be tackled within a city. In this way, the citizens should be enabled to share their ideas in the decision-making process. Consequently, they get full access to the public open data of their cities and can give feedback on neighbourhood-related and citywide ideas. The platform allows governments, NGOs, businesses and citizens alike to configure 
their own topics. As a result, the citizens are empowered to play an active role in the public domain.

\subsubsection{Application design and Implementation}

smarticipate is designed as a generic web-based application which lets the administrator of the platform configure so called Topics. Those Topics can be anything from bike rack planning questions, to finding places for urban gardening or letting citizens decide on future uses of buildings in a refurbishment area. Those Topics will then be configured in the platform's back-end. Additionally, to the usual configuration features of a topic (name, description) smarticipate provides unique functions: (i) the definition of (generic) objects to be placed on the topic's map (here: Tree types, Figure 6) resembles a portion of the back-end's Topic configuration interface.

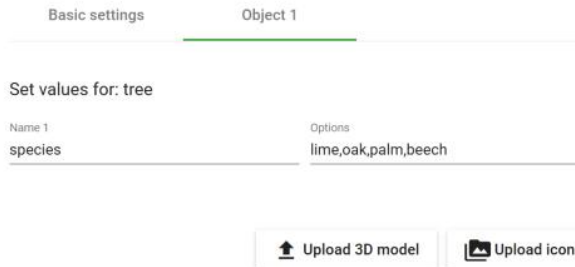

Figure 6. smarticipate Back-End - Object Configuration (here: Trees) (c) AIT 2018

(ii) the upload of user e-mail addresses who want to/shall be contacted/invited regarding the specific Topic in question (Figure 7 , button on the left hand side)

(iii) the upload of so called rules to be used to give direct feedback on locations of the (above configured) placeable objects when clicking the map. The rules are used for querying the above mentioned OGD datasets. (Figure 7, button in the middle) (iv) additionally, an area of interest (AOI) for the Topic in question (e.g. a district or a neighbourhood within a city) and background maps (depicting the current situation of a Topic) can be configured (Figure 7, button on the left-hand side and at the bottom of the page). These background maps are directly loaded from the city's geo-server and could e.g. depict the current land use, legal restrictions or locations of bus stations, lamp poles etc., within the AOI. The links to those maps can be easily configured via Web Map Service (WMS) links to the city's OGD server.

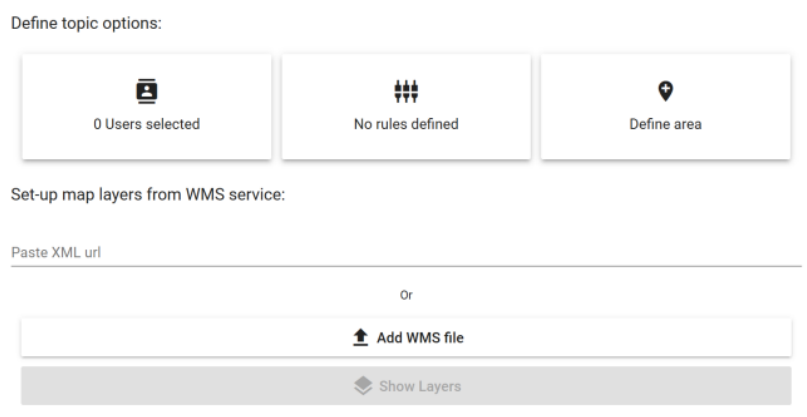

Figure 7. smarticipate Back-End - User Invitation and Rules Configuration Interface () AIT 2018

The configured Topic is published on the smarticipate's frontend main page and can be opened by the citizens, either in a desktop or a mobile browser. The configured objects can be chosen on the right-hand side of the interface (Figure 8) and can then be placed on the map as a proposal. When doing so, the system triggers a rule query to the underlying OGD dataset(s) which then leads to a feedback to the user if placing the object at this location is possible and if not, why it is not possible. It is important to notice that even if the location indicates that placing an object is not possible, the user can still propose the location for further discussion with other citizens or the city administration to discuss a change in the legal or planning prerequisites for this neighbourhood.

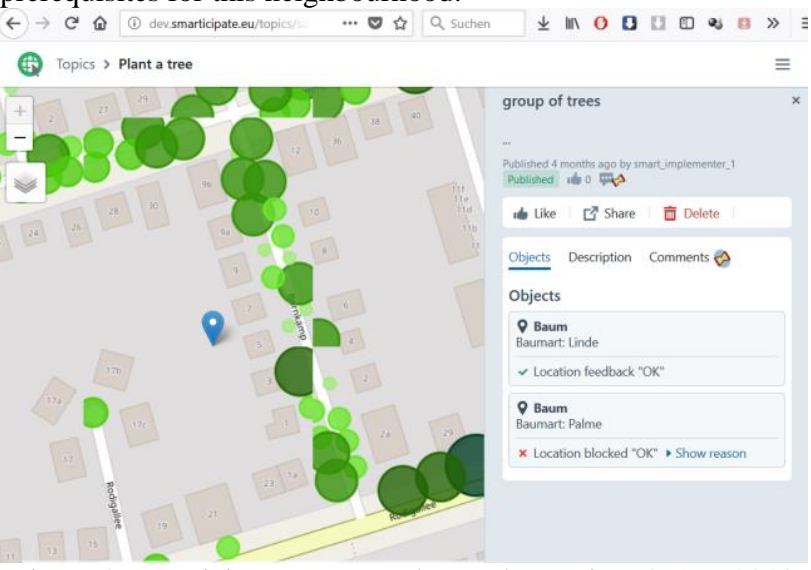

Figure 8. smarticipate Front-End - Desktop View (C) AIT 2018

Another feature of the platform is its commenting and voting function: The users can comment on other users' proposals and vote for them in the right hand side application bar of the interface, in this way fostering an active discussion on neighbourhood issues.

Additionally to the above described features, smarticipate also provides the possibility to depict 3D scenes of objects covered by the Topic (where CityGML $\left({ }^{3}\right)$, a data format to store city related data including 3D information, is available). In this way it is possible to also discuss design issues of buildings, trees or any other kind of physical object in the AOI of the Topic (Figure 9).

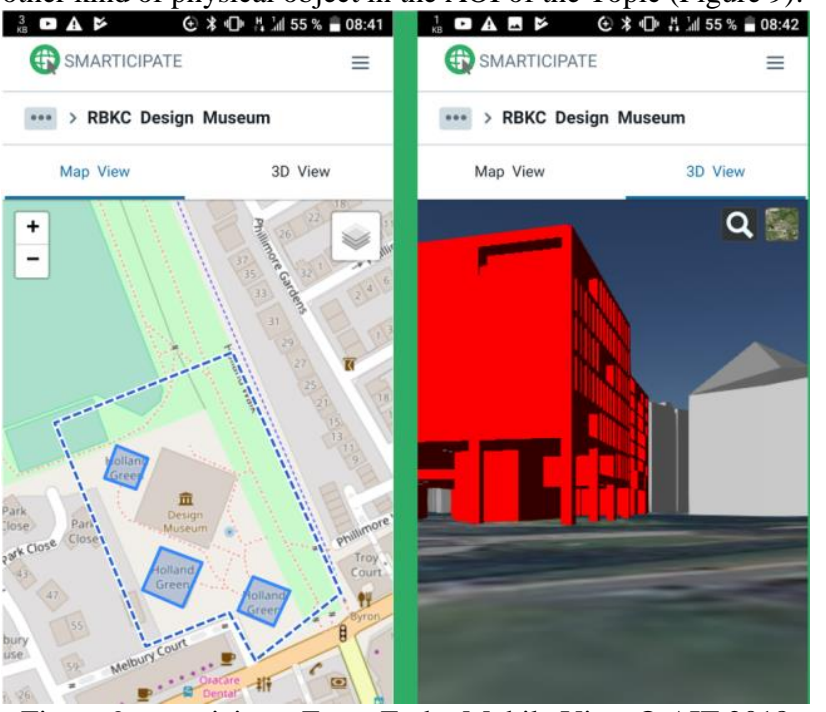

Figure 9. smarticipate Front-End - Mobile View (C) AIT 2018

\subsubsection{Difficulties and possible solutions}

Since smarticipate's unique feature is the integration of OGD with feedback functionalities based on defined rules, it is always necessary to get domain experts' advice from within the city administration. Those experts need to provide background information to be able to describe the rules necessary for the discussion of a Topic. During the development phase of the

\footnotetext{
${ }^{3}$ https://www.citygml.org/, last accessed: 15.05.2018
} 
platform this proved to be an important, yet quite complex task: Planners and civil servants needed to understand the idea behind the feedback system in order to provide the necessary information. Often the rule information (coming in form of e.g. tabular matrices) were not sufficient to depict the complexity of the Topic to the citizens. Here, a lot of effort is needed to translate e.g. legal planning rules into digestible feedback for the citizens. Possible solutions for this problem have also been developed within the project: The methodology is called smartathon. This gathering of citizens with their administration, which have been attended by around 100 people during the development phase of the platform, have been used to discuss the platforms features and usability aspects. In the future, those smartathons will also be used to discuss planning topics in a physical meeting which can then be translated into rules for the system to be used by a broader public within the smarticipate platform.

\section{Discussion}

In recent years, more and more spatial web-applications have emerged, that allow the user not only to look at static, precalculated results, but also to perform interactive analyses that deliver exactly the results that are of interest to the user. The presented case studies thus enhance the standard approach for geo-visualization.

The reflection of the three different applications shows some of the main challenges in the development of interactive spatial web-applications. For example, the advantage of using the $\mathrm{R} /$ Shiny framework is the possibility to develop applications that are easy to understand and relatively easy to develop with low coding effort. The interactive usage fosters urban co-creation and citizens - as local experts - can easily develop different scenarios and learn from the (simulated) effects on the urban environment. Furthermore, due to the circumstances that the results can thus be more tailored to the user's needs - e.g., results for the local planning processes - is the public engagement easier to achieve. The two presented R/Shiny applications have in common that compared to professional software or games - the development effort is low and user experience testing is not very elaborated. Additionally due to the use of predefined frameworks as R/Shiny also drawbacks regarding performance and design have to be accepted. Nevertheless, the use of simple online Help-Systems (Tooltips, annotated screenshots and tutorial videos etc.) can reduce these negative effects.

On the other hand, the smarticipate portal's front-end is programmed in ReactJS which is a modern framework for programming device aware web-sites which adapt to different screen sizes easily. The use of maps in the platform is greatly improved by using this framework, still, even with this modern framework, the map depiction and usage of markers e.g. is not optimal if used below the screen size of a tablet. This again shows that geo-visualisations on screen sizes of mobile phones are a very challenging task which needs a lot of design considerations before the actual programming can take place.

\section{CONCLUSION}

This paper described how interactive spatial web-applications can be used to support local stakeholders and urban planners in spatial decision making by implementing citizen's thoughts and OGD datasets.

In the future most likely more spatial interactive webapplications will be developed as their potential support for participatory urban planning processes is very high. Most of all, because the development of these applications can now also be done by non-professional developers, like data scientists who have already tools available to create useful applications for many different topics.

Regarding the smarticipate platform it can be expected that more and more generic platforms will emerge in the future which are created in a single big development effort but deliver generic configuration capabilities to reduce the complexity of programming even further. This will allow also for lay people to create relevant topics for their neighbourhoods and enable them to participate more actively in the decision-making processes of their cities. As a conclusion it can be expected that participation will become more attractive for citizens since the barriers to do so will be lowered by those applications and with the next generations of younger, digitally more elaborated users the acceptance of digital tools like the ones described here will further increase.

\section{ACKNOWLEDGEMENTS}

Syn[En]ergy acknowledges the funds in the frame of the $4^{\text {th }}$ "Stadt der Zukunft" - program, from the Austrian Ministry for Transport, Innovation and Technology coordinated by the Austrian Research Promotion Agency (FFG), the national funding agency for industrial research and development in Austria.

Memphis acknowledges the funds of IEA DHC - the International Energy Agency Technology Collaboration Programme on District Heating and Cooling including Combined Heat and Power.

Project smarticipate has received funding from the European Union's Horizon 2020 research and innovation programme 2016 - 2019 under grant agreement No 693729.

\section{REFERENCES}

Al-Yafi, K., El-Masri, M., 2016. Gamification of e-Government Services: A Discussion of Potential Transformation, in: Twenty-Second Americas Conference on Information Systems. San Diego, p. 9.

Barata, G., Gama, S., Jorge, J., Gonçalves, D., 2013. Improving Participation and Learning with Gamification, in: Proceedings of the First International Conference on Gameful Design, Research, and Applications, Gamification '13. ACM, New York, NY, USA, pp. 10-17.

https://doi.org/10.1145/2583008.2583010

Cayola, L., Macías, J.A., 2018. Systematic guidance on usability methods in user-centered software development Information and Software Technology 97, 163-175. https://doi.org/10.1016/j.infsof.2018.01.010

Coronado Escobar, J.E., Vasquez Urriago, A.R., 2014. Gamification: An Effective Mechanism to Promote Civic Engagement and Generate Trust?, in: Proceedings of the 8th International Conference on Theory and Practice of Electronic Governance, ICEGOV'14. ACM, New York, NY, USA, pp. 514-515. https://doi.org/10.1145/2691195.2691307

DeCodingSpaces Toolbox [WWW Document], 2018. URL https://decodingspaces-toolbox.org/ (accessed 7.14.18).

Desouza C., Kevin, Bhagwatwar, Akshay, 2012. Citizen Apps to Solve Complex Urban Problems, Journal of Urban Technology, Vol. 19, No. 3. 
Deterding, S., Dixon, D., Khaled, R., Nacke, L., 2011. From Game Design Elements to Gamefulness: Defining "Gamification" 7.

Dörk, M., Monteyne, D., 2011. Urban Co-Creation: Envisioning New Digital Tools for Activism and Experimentation in the City. CHI 20114.

Fernandez, A., Insfran, E., Abrahão, S., 2011. Usability evaluation methods for the web: A systematic mapping study. Information and Software Technology 53, 789-817. https://doi.org/10.1016/j.infsof.2011.02.007

Fowler, S.L., Stanwick, V.R., 2004. Web application design handbook: best practices for web-based software, The Morgan Kaufmann series in interactive technologies. Morgan Kaufmann Publishers, Amsterdam; Boston.

GDAL/ORG contributors, 2018. GDAL: GDAL - Geospatial Data Abstraction Library [WWW Document]. URL http://www.gdal.org/ (accessed 5.9.18).

Gebetsroither-Geringer, E., Loibl, W., 2014. Urban Development Simulator: An Interactive Decision Support Tool for Urban Planners Enabling Citizen's Participation.

IBE Software, 2018. HelpNDoc | HTML help authoring tool, Word, PDF and eBook documentation generator [WWW Document]. URL https://www.helpndoc.com/ (accessed 5.12.18).

Kazhamiakin, R., Marconi, A., Perillo, M., Pistore, M., Valetto, G., Piras, L., Avesani, F., Perri, N., 2015. Using gamification to incentivize sustainable urban mobility, in: 2015 IEEE First International Smart Cities Conference (ISC2). Presented at the 2015 IEEE First International Smart Cities Conference (ISC2), pp. 1-6. https://doi.org/10.1109/ISC2.2015.7366196

Khan, Z., Ludlow, D., Loibl, W., Soomro, K., 2014. ICT enabled participatory urban planning and policy development: The UrbanAPI project. Transforming Government: People, Process and Policy 8, 205-229. https://doi.org/10.1108/TG-092013-0030

Lim, A., Tjhi, W., 2015. R high performance programming. Overcome performance difficulties in $\mathrm{R}$ with a range of exciting techniques and solutions. Packt Publishing, Birmingham und Mumbai.

Lovelace, R., Nowosad, J., Muenchow, J., 2018.

Geocomputation with R.

Martin, 2016. "Benchmark: R vs. Python Rasterio vs. GDAL" is locked Benchmark: R vs. Python Rasterio vs. GDAL | GISBlog.com.

Matera, M., Rizzo, F., Carughi, G.T., 2006. Web usability: principles and evaluation methods, in: In E. Mendes and N. Mosley (Eds), Web Engineering (Pp. Springer Verlag, pp. 143180.

Mueller, J., Lu, H., Chirkin, A., Klein, B., Schmitt, G., 2018. Citizen Design Science: A strategy for crowd-creative urban design, Cities 72 (2018).

Mvungi, J., Tossy, T., 2015. Usability Evaluation Methods and Principles for the Web. International Journal of Computer Science and Software Engineering (IJCSSE) 4, 7.
Nielsen, Jakob, 2012. Usability 101: Introduction to Usability. Prat, 2018. Top 8 Challenges in Web Application Development in 2018 .

R Foundation, 2018. R: The R Project for Statistical Computing [WWW Document]. URL https://www.r-project.org/ (accessed 5.9.18).

R-Studio, 2018. Shiny [WWW Document]. URL https://shiny.rstudio.com/ (accessed 5.9.18).

Shahzad, F., 2017. Modern and Responsive Mobile-enabled Web Applications. Procedia Computer Science, 14th International Conference on Mobile Systems and Pervasive Computing (MobiSPC 2017) / 12th International Conference on Future Networks and Communications (FNC 2017) / Affiliated Workshops 110, 410-415.

https://doi.org/10.1016/j.procs.2017.06.105

Stadt Wien, 2018. Sag's Wien - Die App für Ihre Anliegen an die Stadt [WWW Document]. URL

https://www.wien.gv.at/sagswien/index.html (accessed 5.15.18).

Sugumaran, V., Sugumaran, R., 2007. Web-based Spatial Decision Support Systems (WebSDSS): Evolution,

Architecture, Examples and Challenges (No. Volume 19), Communications of the Association for Information Systems.

Thiel, Sarah-Kristin, Lehner, Ulrich, 2015. Exploring the effects of game elements in m-participation [WWW Document]. ResearchGate. URL

https://www.researchgate.net/publication/300204780_Exploring _the_effects_of_game_elements_in_m-participation (accessed 5.13.18).

UrbanFootprint [WWW Document], 2018. . UrbanFootprint. URL https://urbanfootprint.com/ (accessed 7.14.18).

UrbanSim Inc [WWW Document], 2018. . UrbanSim. URL http://www.urbansim.com/ (accessed 7.14.18).

URENIO Research, 2018. Smart City Applications - Improve My City [WWW Document]. URL

http://smartcityapps.urenio.org/improve-my-city_en.html (accessed 5.15.18).

Van Ransbeeck, Wietse, 2016. Gamification in Citizen Participation. CitizenLab.

Voss, A., Denisovich, I., Gatalsky, P., Gavouchidis, K., Klotz, A., Roeder, S., Voss, H., 2004. Evolution of a participatory GIS. Computers, Environment and Urban Systems, AGILE 2003 28, 635-651.

https://doi.org/10.1016/j.compenvurbsys.2003.12.003

Wiener Umweltschutzabteilung, 2018. Wien Umweltgut - der Themenstadtplan zu umweltrelevanten Informationen in Wien [WWW Document]. URL

https://www.wien.gv.at/umweltschutz/umweltgut/ (accessed 5.15.18).

Wroblewski, L., Rantanen, E.M., 2001. Design Considerations for Web-Based Applications. Proceedings of the Human Factors and Ergonomics Society Annual Meeting 45, 1191-1195. https://doi.org/10.1177/154193120104501508 